American Journal of Agricultural and Biological Sciences 2 (4): 260-266, 2007

ISSN 1557-4989

(C) 2007 Science Publications

\title{
Nucleation and Crystal Growth Rates of Struvite in DTM Type Crystallizer with a Jet- Pump of Descending Suspension Flow in a Mixing Chamber
}

\author{
${ }^{1}$ Joanna Koralewska, ${ }^{2}$ Krzysztof Piotrowski, ${ }^{1}$ Boguslawa Wierzbowska and ${ }^{1}$ Andrzej Matynia \\ ${ }^{1}$ Faculty of Chemistry, Wroclaw University of Technology, \\ Wybrzeze Wyspianskiego 27,50-370 Wroclaw, Poland \\ ${ }^{2}$ Department of Chemical and Process Engineering, Silesian University of Technology, ks. M. Strzody 7 , \\ 44-101 Gliwice, Poland
}

\begin{abstract}
The kinetic data concerning reaction-crystallization of struvite from diluted water solutions of $\mathrm{Mg}^{2+}, \mathrm{NH}_{4}{ }^{+}$and $\mathrm{PO}_{4}{ }^{3-}$ ions in a continuous DTM-type liquid jet-pump crystallizer with internal circulation of suspension are presented and discussed. The measurements were performed with the assumption of minimal value of unit power of a jet-pump's feeding stream, providing thus a minimal level of internal circulation intensity. Nucleation and linear growth rates of struvite crystals were calculated on the basis of population density distributions of product particles. A mathematically convenient kinetic model for MSMPR crystallizer, making allowance for a size-dependent growth (SDG) phenomenon, was adopted for description of a complex process of precipitation integrated with crystals growth. It was concluded, that in the concentration range of magnesium ions in a feeding solution $\left[\mathrm{Mg}^{2+}\right]_{\mathrm{RM}}=0.1-2.0$ mass $\%$ and, in stoichiometric proportions, phosphate ions $\left[\mathrm{PO}_{4}{ }^{3-}\right]_{\mathrm{RM}}=$ $0.39-7.81$ mass \% as well as the ammonium ions $\left[\mathrm{NH}_{4}{ }^{+}\right]_{\mathrm{RM}}=0.074-1.48$ mass $\%$, the Rojkowski hyperbolic SDG kinetic model proved to be an equation the best statistically fitted to the own experimental data.
\end{abstract}

Key words: struvite, reaction-crystallization, SDG MSMPR kinetic model, phosphate removal, phosphorus recycling.

\section{INTRODUCTION}

In modern, advances technologies of effective phosphorus recycle ${ }^{[1-3]}$ elimination of phosphate ions from industrial or municipal liquid wastes, sewage or agricultural manure results from their precipitation in a chemically transformed form of sparingly soluble, complex inorganic salt $-\mathrm{MgNH}_{4} \mathrm{PO}_{4} \cdot 6 \mathrm{H}_{2} \mathrm{O}-$ magnesium / ammonium orthophosphate hexahydrate, MAP, struvite $\left(\mathrm{p} K_{\mathrm{sp}}=9.4-13.26\right)^{1,4]}$. This complex reaction-crystallization process of struvite synthesis is usually run in alkaline environment $(7<\mathrm{pH}<11)$, the most frequent in environment temperature $(T=298 \mathrm{~K})$, feeding the continuous crystallizer with preliminary purified and appropriately diluted phosphate-rich liquid wastes $\left(\mathrm{PO}_{4}{ }^{3-}\right)$, water solution of e.g. magnesium chloride $\left(\mathrm{Mg}^{2+}\right)$ and some ammonium salt $\left(\mathrm{NH}_{4}{ }^{+}\right)$ solution. Despite that at present day these complex processes are not fully explained, technically mastered and commonly used in a large scale, they present an attractive (both technically and economically) alternative in relation to biological methods of phosphorus removal from sewage. Additional benefit of this compact technology is that, aside from equally important sewage purification effects, a valuable crystalline product is obtained, considered to be an more and more important source of recycled phosphorus (as well as ammonium and magnesium), convenient for further processing and/or agricultural application, for example in a form of slowly dissolved (thus having an long-term nutritive effect) mineral fertilizer ${ }^{[5-7]}$.

In order to improve the competitiveness of this method an important problem to solve is to optimally match the crystallizer construction with a set of required technological parameter values. Owing to a complexity of the system under study (e.g. effects of: temperature, $\mathrm{pH}$, synergistic/antagonistic effects between ions and impurities, hydrodynamics of twophase suspension, etc.) the laboratory research is practically the only reliable method to provide the kinetic model of the process with appropriate parameter values. The influence of reagents concentrations, $\mathrm{pH}$ and suspension residence time on the kinetics of

Corresponding Author: Joanna Koralewska, Faculty of Chemistry, Wroclaw University of Technology, Wybrzeze Wyspianskiego 27, 50-370 Wroclaw, Poland 
reaction-crystallization process of struvite synthesis through its continuous precipitation in a DT (Draft Tube) type crystallizer with a propeller agitator was discussed in detail in other authors' work ${ }^{[8]}$. The experimental results of reaction-crystallization of struvite from diluted water solutions of $\mathrm{Mg}^{2+}, \mathrm{NH}_{4}{ }^{+}$and $\mathrm{PO}_{4}{ }^{3-}$ ions in other type of apparatus - a continuous DTM (Draft Tube Magma) type crystallizer with internal circulation of suspension resulting from application of a liquid jet-pump ${ }^{[9]}$ of descending flow of crystal magma in a mixing chamber are presented below. The experiments were run assuming minimal value of unit power of a jet-pump's feeding stream. On the basis of population density distribution of product crystals the kinetic parameters of struvite nucleation and crystal growth were calculated. Kinetic model of an MSMPR (Mixed Suspension Mixed Product Removal) crystallizer, taking under consideration a sizedependent growth (SDG) phenomenon ${ }^{[10,}{ }^{11]}$, was adopted.

The jet-pump constructions can be generally classified as the DTM-type crystallizers ${ }^{[10]}$. If a jetpump device situated inside the crystallizer's vessel guarantees good mixing of the circulated suspension, thus enables one to receive a non-classified product of representative crystal size distribution (CSD), it may be approximately assumed that such apparatus fulfills the MSMPR crystallizer requirements. Its main advantage is an absence of moving (rotating) elements, what brings about a low tendency to failure with relative simplicity in use (see Fig. 1). The jet-pump crystallizers have proved their usefulness for various mass crystallization processes ${ }^{[9,10]}$, including the most complex reaction-crystallization ones. Currently the laboratory tests of their technological applicability in reaction-crystallization of: calcium phosphate $\mathrm{Ca}_{5}\left(\mathrm{PO}_{4}\right)_{3} \mathrm{OH}$ (hydroxyapatite ${ }^{[12,13]}$, barium sulphate $\mathrm{BaSO}_{4}^{[14,15]}$ and struvite ${ }^{[12,16]}$ are performed.

\section{MATERIALS AND METHODS}

Chemicals: For reaction-crystallization of struvite, according to the presented ionic reaction stoichiometry, Eq. (1):

$\mathrm{Mg}^{2+}+\mathrm{NH}_{4}^{+}+\mathrm{H}_{2} \mathrm{PO}_{4}^{-}+6 \mathrm{H}_{2} \mathrm{O}=\mathrm{MgNH}_{4} \mathrm{PO}_{4} \cdot 6 \mathrm{H}_{2} \mathrm{O}+2 \mathrm{H}^{+}$

the following reagents were applied:

- Magnesium chloride hexahydrate, $\mathrm{MgCl}_{2} \cdot 6 \mathrm{H}_{2} \mathrm{O}$, p.a., POCh Gliwice (Poland),

- Ammonium dihydrogenphosphate, $\mathrm{NH}_{4} \mathrm{H}_{2} \mathrm{PO}_{4}$, p.a. (POCh Gliwice, Poland).
Selected physical properties of these substances are presented by Mullin ${ }^{[17]}$.

The reaction environment's $\mathrm{pH}$ was adjusting by addition of sodium hydroxide, $\mathrm{NaOH}$, p.a. (POCh Gliwice, Poland).

Experimental setup and procedure: The simplified scheme of laboratory stand with an liquid jet-pump DTM-type crystallizer providing descending flow of suspension in a mixing chamber during its internal circulation (denoted later as a DTM $\downarrow$ construction) is presented in Fig. 1. Integrated automatic control / adjustment of experimental plant operation, as well as experimental data recording, were done with the use of PC computer. The simplified scheme of a liquid jetpump applied is presented in Fig. 2. Geometrical proportions of its individual constructional elements, dimensions, as well as its location inside the crystallizer vessel were rationally selected on the basis of the initial test results ${ }^{[18]}$.

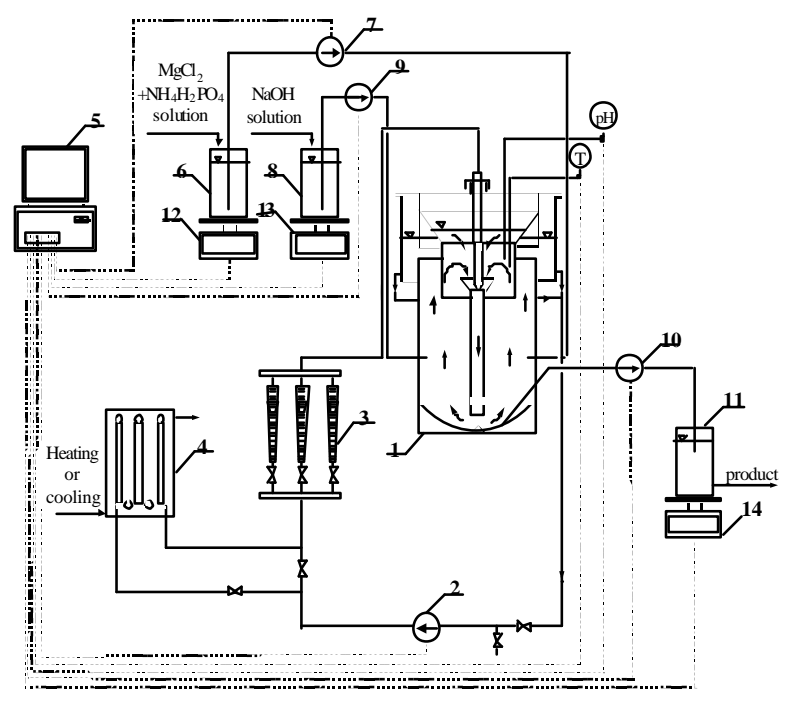

Fig. 1: Experimental laboratory stand scheme: 1 - DTM $\downarrow$ crystallizer with internal circulation of suspension of working volume $\mathrm{Vw}=1.2 \mathrm{dm} 3$ (geometrical proportions: diameter $\mathrm{D}=90 \mathrm{~mm}$, height $\mathrm{H}=330 \mathrm{~mm}$ ), 2 - external circulation pump, 3 - set of flow rotameters, 4 - set of heat exchangers, 5 - PC computer (central monitoring and process control), 6 - feeding solution tank $(\mathrm{MgCl} 2$ water solution initially blended with water solution of NH4H2PO4), 7 - peristaltic dosing pump, 8 - alkaline agent tank: $\mathrm{NaOH}$ solution, $9-\mathrm{NaOH}$ dosing pump (peristaltic), 10 - pump for removal of struvite crystal product suspension from the crystallizer vessel, 11 transitional storage tank for struvite crystal product suspension, $12,13,14$ - precise electronic balances, $\mathrm{pH}-$ control of the reaction environment's $\mathrm{pH}, \mathrm{T}$ - temperature control. 


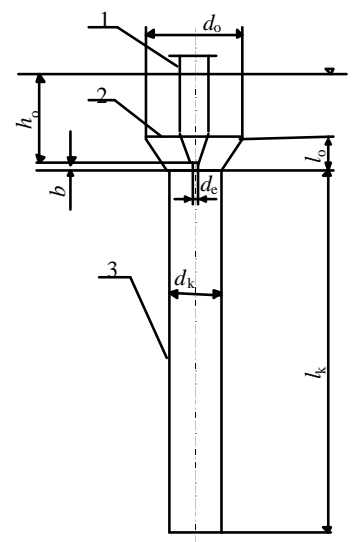

Fig. 2: Liquid-liquid jet-pump device applied in a laboratory DTM $\downarrow$ crystallizer under study: 1 - feeding nozzle, 2 confuzor, 3 - mixing chamber.

The crystallizer was fed with a water solution of magnesium chloride and - in a stoichiometric proportion - ammonium dihydrogenphosphate(V) (of concentration 25 mass $\left.\% \mathrm{NH}_{4} \mathrm{H}_{2} \mathrm{PO}_{4}\right)$. Both solutions were initially effectively blended in a feeding solution tank (see Fig. 1). The resulting mixture of reaction substrates (of strictly controlled concentration of ion compounds) was introduced into so-called "crystal growth zone" in a crystallizer (occupying a ring-form space between a crystallizer body and a jet-pump's mixing chamber). The zone was also provided with water solution of sodium hydroxide (of concentration 20 mass \% $\mathrm{NaOH}$ ), in appropriate proportion, warranting the required value of the environment's $\mathrm{pH}$ necessary for struvite precipitation process. The feeding points and location of the product crystals suspension removal are marked in Fig. 1. A jet-pump's feeding nozzle was installed below upper level of medium in the crystallizer.

All measurements were performed in a constant temperature, $T=298 \mathrm{~K}$ at $\mathrm{pH}=9$ and providing average residence time of suspension $\tau=900 \mathrm{~s}$. Concentration of magnesium ions in a feeding solution was changed from 0.1 up to 2.0 mass $\%$. Concentrations of other ions, necessary for MAP synthesis and precipitation, were precisely adjusted within the appropriate stoichiometric proportions: phosphate ones - from 0.39 to 7.81 mass \% and ammonium ones from 0.074 to 1.48 mass $\%$.

The crystallizer was operated assuming a minimal value of unit power of feeding stream, $P_{\mathrm{eu}}{ }^{[19]}, \mathrm{Eq}$. (2):
$P_{\mathrm{eu}}=p_{\mathrm{de}} \mathrm{q}_{\mathrm{ve}}=\frac{?_{\mathrm{sol}} v_{\mathrm{e}}^{2} \mathrm{q}_{\mathrm{ve}}}{2 ?_{\mathrm{sus}} V_{\mathrm{w}}}=\frac{8 ?_{\mathrm{sol}} q_{\mathrm{ve}}^{3}}{\mathrm{p}^{2} d_{\mathrm{e}}^{4} ?_{\mathrm{sus}} V_{\mathrm{w}}}$

Where: $P_{\text {eu }}-$ unit power of a feeding liquid (clarified) stream $\left(\mathrm{W} \mathrm{kg}^{-1}\right) ; p_{\mathrm{de}}-$ dynamic pressure of a feeding stream $(\mathrm{Pa}) ; q_{\mathrm{ve}}-$ volumetric flow rate of a feeding stream $\left(\mathrm{m}^{3} \mathrm{~s}^{-1}\right) ; \rho_{\mathrm{sol}}-$ solution density $\left(\mathrm{kg} \mathrm{m}^{-3}\right) ; v_{\mathrm{e}}-$ linear velocity of a feeding stream $\left(\mathrm{m} \mathrm{s}^{-1}\right) ; \rho_{\text {sus }}-$ suspension density $\left(\mathrm{kg} \mathrm{m}^{-3}\right) ; V_{\mathrm{w}}$ - crystallizer's working volume $\left(\mathrm{m}^{3}\right) ; d_{\mathrm{e}}-$ feeding nozzle's diameter $(\mathrm{m})-$ see Fig. 2.

The jet-pump's feeding nozzle was thus provided with a minimum volumetric flow rate of a circulated stream, indispensable only for keeping all particles within a crystallizer in a continuous rotating movement. Circulation intensity was thus minimal, too.

The continuous, integrated reaction-crystallization process was run through the time $5 \tau$ (beginning from the moment of the assumed process parameter values stabilized). After this time, the solid phase content in the product crystal suspension $\left(M_{\mathrm{T}}\right)$ and its crystal size distribution (CSD) were established. Analysis of mother liquor (plasma emission spectrometer ICP AES PHILIPS PU 7000), solid phase compositions (spectrometer IR PHILIPS PU 9712) and volumetric / mass CSD (laser particle size analyzer COULTER LS 230) were performed. Crystal images were made with the use of scanning electron microscope JEOL JSM 5800LV.

From the mass (or volumetric) crystal size distribution of the MAP product withdrawn from the crystallizer, the population density values, $n_{\mathrm{i}}$, were calculated, Eq. (3):

$n_{\mathrm{i}}=\frac{m_{\mathrm{i}}}{k_{\mathrm{v}} ? L_{\mathrm{i}}^{3} ? L_{\mathrm{i}} V_{\mathrm{w}}}=\frac{V_{\mathrm{i}}}{k_{\mathrm{v}} L_{\mathrm{i}}^{3} ? L_{\mathrm{i}} V_{\mathrm{w}}}$

Where: $n_{\mathrm{i}}$ - population density of $i-$ th crystal fraction $\left(\mathrm{m}^{-1} \mathrm{~m}^{-3}\right) ; m_{\mathrm{i}}-$ mass of $\mathrm{i}$-th crystal fraction $(\mathrm{kg}) ; k_{\mathrm{v}}-$ crystal's volumetric shape factor $(-) ; L_{\mathrm{i}}-$ mean size of i-th crystal fraction $(\mathrm{m}) ; \Delta L_{\mathrm{i}}-$ size range of $\mathrm{i}-$ th crystal fraction $(\mathrm{m}) ; V_{\mathrm{w}}$ - crystallizer's working volume $\left(\mathrm{m}^{3}\right)$; $V_{\mathrm{i}}$ - volume of i-th crystal fraction $\left(\mathrm{m}^{3}\right)$.

\section{RES ULTS AND DISCUSSION}

Despite low intensity of suspension circulation in a DTM $\downarrow$ crystallizer (since a minimum $P_{\text {eu }}$ value was 
intentionally used), thus the possibility of relatively high values of local supersaturation appearance, the product crystals of mean size from 15.6 to $31.0 \mu \mathrm{m}$ (see Tab. 1) were obtained. Only insignificant results of agglomeration processes (see Fig. 3) were reported. For comparison purposes it should be stated, that providing intensive mixing in a DT-type crystallizer with propeller agitator, assuming identical values of all other process parameters (concentrations, flow rates, $\mathrm{pH}$, temperature), the struvite crystals of mean size between $10-15 \mu \mathrm{m}$ only were $\operatorname{produced}^{[20]}$.

Table 1: Kinetic parameter values of struvite nucleation and crystal growth in a liquid-liquid jet-pump DTM $\downarrow$ crystallizer (parameter values estimated on the basis of Rojkowski hyperbolic SDG kinetic model, Eq. (4)).

\begin{tabular}{cccccccc}
\hline$\left[\mathrm{Mg}^{2+}\right]_{\mathrm{RM}}$ & $\mathrm{P}_{e u}$ & $L_{m}$ & $G_{0}$ & $G_{8}$ & $a$ & $n_{0}$ & $B$ \\
\hline mass $\%$ & $\mathrm{~W} \mathrm{~kg}^{-1}$ & $\mu \mathrm{m}$ & $\mathrm{m} \mathrm{s}^{-1}$ & $\mathrm{~m} \mathrm{~s}^{-1}$ & $\mathrm{~m}^{-1}$ & $\mathrm{~m}^{-1} \mathrm{~m}^{-3}$ & $\mathrm{~m}^{-3} \mathrm{~s}^{-1}$ \\
\hline 0.1 & 0.11 & 15.6 & $3.74 \cdot 10^{-10} 3.48 \cdot 10^{-8} 9894$ & $4.15 \cdot 10^{21}$ & $1.55 \cdot 10^{12}$ \\
0.25 & 0.20 & 15.5 & $3.33 \cdot 10^{-10} 6.82 \cdot 10^{-8} 5244$ & $1.28 \cdot 10^{22}$ & $4.27 \cdot 10^{12}$ \\
0.5 & 0.24 & 22.5 & $2.32 \cdot 10^{-10} 6.82 \cdot 10^{-8} 6870$ & $4.83 \cdot 10^{22}$ & $1.12 \cdot 10^{13}$ \\
1.0 & 0.45 & 27.9 & $1.65 \cdot 10^{-10} 1.13 \cdot 10^{-7} 4190$ & $5.33 \cdot 10^{22}$ & $8.81 \cdot 10^{12}$ \\
2.0 & 1.45 & 31.0 & $1.79 \cdot 10^{-10} 5.02 \cdot 10^{-7} 1003$ & $9.48 \cdot 10^{22}$ & $1.69 \cdot 10^{13}$ \\
\hline
\end{tabular}

for $k_{\mathrm{v}}=1, T=298 \mathrm{~K}, \mathrm{pH}=9, \tau=900 \mathrm{~s}$

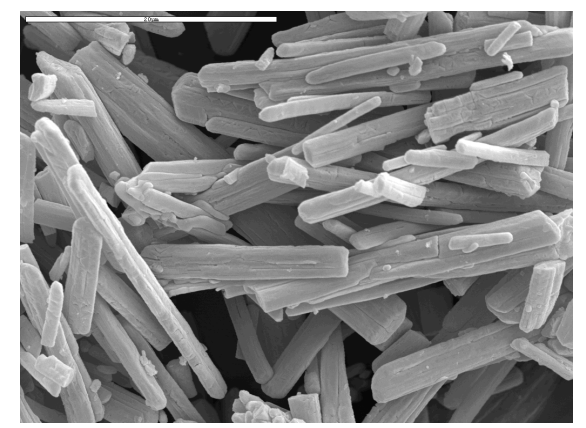

Fig. 3: Scanning electron microscope image of struvite crystals produced in a laboratory DTM $\downarrow$ crystallizer. The process parameters assumed: $\left[\mathrm{Mg}^{2+}\right]=1.0$ mass $\%,\left[\mathrm{PO}_{4}{ }^{3-}\right]_{\mathrm{RM}}=3.91$ mass $\%,\left[\mathrm{NH}_{4}{ }^{+}\right]_{\mathrm{RM}}=0.74$ mass $\%, T=298 \mathrm{~K}, \mathrm{pH}=9, \tau=$ 900 s. Magnification: 3000x.

As it can be concluded from Tab. 1, in a DTM $\downarrow$ crystallizer the mean crystal size of struvite crystals increases with the increase of magnesium (thus proportionally - also with ammonium and phosphate) ions concentrations in a feeding solution, in spite of simultaneous increase of solid phase concentration in a circulated suspension (suspension density, $M_{\mathrm{T}}$ ) from 10 to $195 \mathrm{~kg}$ crystals $\mathrm{m}^{-3}{ }^{[12]}$. Exemplary population density distributions of struvite product crystals withdrawn from a DTM $\downarrow$ crystallizer ${ }^{[21]}$ are presented in Fig. 4.

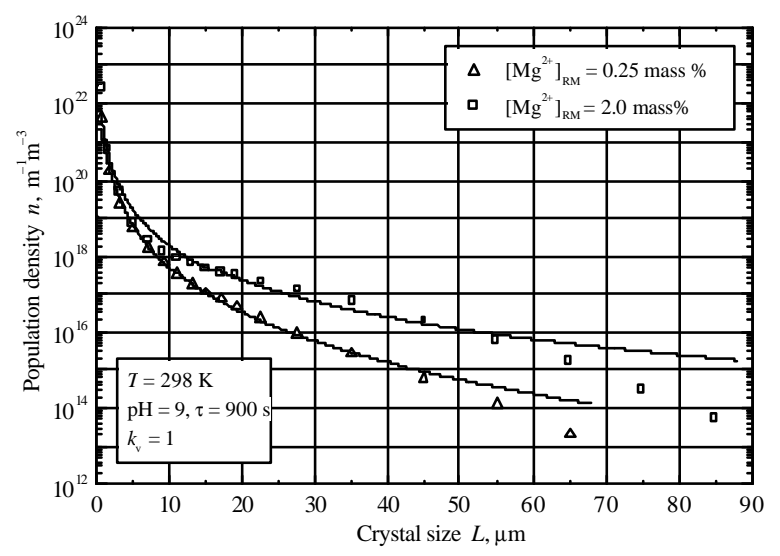

Fig. 4: Comparison of population density values of struvite crystals: individual points - experimental data, solid lines - the values calculated with Eq. (4) and kinetic data from Tab. 1 (Rojkowski hyperbolic SDG model applied) for the two selected values of magnesium ions concentration in a feeding solution, $\left[\mathrm{Mg}^{2+}\right]_{\mathrm{RM}}=0.25$ and 2.0 mass $\%$.

From the plot it results, that for the crystals of relatively small sizes (below ca. $10 \mu \mathrm{m}$ ) the crystal population density distribution presented in a $\ln n-L$ coordinate system deviates towards top. Thus, the data course suggests an occurrence in the system studied a complex kinetic phenomenon - size-dependent growth rate $^{[10]}$ (SDG), which must be encountered in the precise model of this process. Using statistical methods (nonlinear regression) an appropriate kinetic SDG model was thus selected (assumed criterion - a minimum value of sum of variances over all $n(L)$ measurement series). Five SDG models were taken under consideration: Canning-Randolph ${ }^{[22]}$, ASL (Abegg-Stevens-Larson) ${ }^{[23]}$ as well as Rojkowski exponential $^{[24]}$, Rojkowski hyperbolic ${ }^{[25]}$ and Rojkowski hyperbolic $\mathrm{II}^{[26]}$ ones. The Rojkowski hyperbolic ${ }^{[25]}$ model proved to be an optimal one in respect to quality of fitting to the own experimental data-set, providing the values of $G_{0}, G_{\infty}$ and $n_{0}$, Eqs. (4) and (5):

$$
\begin{aligned}
& n=n_{0} \exp \left[-\left(\frac{1}{\mathrm{t}} \frac{G_{\infty}-G_{0}}{a G_{\infty}^{2}} \ln \left(\frac{a G_{\infty} L+G_{0}}{G_{0}}\right)+\frac{1}{\mathrm{t}} \frac{L}{G_{\infty}}+\ln \left(\frac{G_{0}+a G_{\infty} L}{(1+a L) G_{0}}\right)\right)\right] \\
& G=G_{\infty}-\left(\frac{G_{\infty}-G_{0}}{1+a L}\right)=\frac{G_{0}+a G_{\infty} L}{1+a L}
\end{aligned}
$$


Where: $n_{0}-$ nuclei (zero-size crystals) population density $\left(\mathrm{m}^{-1} \mathrm{~m}^{-3}\right) ; G_{\infty}-$ maximal (asymptotic) crystal's linear growth rate $\left(\mathrm{m} \mathrm{s}^{-1}\right) ; G_{0}-$ minimal crystal's linear growth rate (growth rate of nuclei) $\left(\mathrm{m} \mathrm{s}^{-1}\right) ; a_{-}$ parameter in a Rojkowski hyperbolic SDG model $\left(\mathrm{m}^{-1}\right)$.

Knowing a nuclei population density value $\left(n_{0}\right)$, as well as their linear growth rate (the minimal one, $G_{0}$ ), the nucleation rate parameter $(B)$ can be directly calculated as (Eq. (6)):

$$
B=n_{0} G_{0}
$$

For the selected concentration values of magnesium ions in a raw material $\left(\left[\mathrm{Mg}^{2+}\right]_{\mathrm{RM}}=0.25\right.$ and 2.0 mass $\%$ ) the $n(L)$ (Eq. (4)) course was presented graphically in Fig. 4. The size-dependence of linear growth rate of struvite crystals, according to a Rojkowski hyperbolic SDG model (Eq. (5)), is presented in Fig. 5.

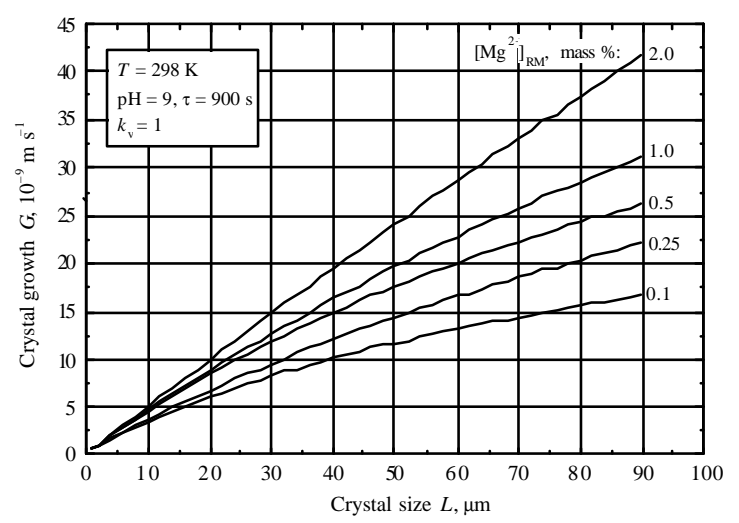

Fig. 5: The size-dependence of linear growth rate of struvite crystals for all five magnesium ions concentrations in a feeding solution tested - Eq. (5) and data from Tab. 1 (Rojkowski hyperbolic SDG model applied).

In Table 1 the Eqs. (4) and (5) parameters values, calculated with the use of nonlinear regression methods, as well as nucleation rate $(B)$ values were presented. From these data it can be concluded, that with the increase of magnesium ions concentration in a feeding solution, $\left[\mathrm{Mg}^{2+}\right]_{\mathrm{RM}}$, from 0.1 up to 2.0 mass $\%$, the required unit power of feeding stream, $P_{\mathrm{eu}}$, increases: from 0.11 up to $1.45 \mathrm{~W} \mathrm{~kg}^{-1}$, what is connected both with the increase of crystal phase concentration in a resulting suspension (discussed above increase of $\mathrm{M}_{\mathrm{T}}$ : $10 \rightarrow 195 \mathrm{~kg} \mathrm{~m}^{-3}$ ), thus its unit weight increase, as well as an increase of $L_{\mathrm{m}}$ parameter value (hydrodynamical effects resulting from dealing with larger individual particles). With $\left[\mathrm{Mg}^{2+}\right]_{\mathrm{RM}}$ parameter value increase in the presented above range, the mean size of crystal product, $L_{\mathrm{m}}$, increases: from $15.6 \mu \mathrm{m}$ to $31.0 \mu \mathrm{m}$. It results from two visible tendences: increase of maximal growth rate for larger crystals, $G_{\infty}$, from $3.48 \cdot 10^{-8} \mathrm{~m} \mathrm{~s}^{-1}$ to $5.02 \cdot 10^{-7} \mathrm{~m} \mathrm{~s}^{-1}$ (more than one magnitude increment), directly responsible for the evaluation of larger particles, as well as from the increasing parallelly - but not able to dominate the whole process kinetics nucleation rate, $B$, from $1.55 \cdot 10^{12} \mathrm{~m}^{-3} \mathrm{~s}^{-1}$ to $1.69 \cdot 10^{13} \mathrm{~m}^{-}$ ${ }^{3} \mathrm{~s}^{-1}$ (about one magnitude increment only). It can be an proof to assume, that in this system the growth rate is more supersaturaton-dependent than nucleation, what is advantageous for large crystals production. From this reason the increase of $\mathrm{Mg}^{2+}$ ions concentration in a feeding stream does not lead to a undesirable, sudden increase in the number of the smallest particles (nuclei) in the system (increment of $\mathrm{n}_{0}: 4.15 \cdot 10^{21} \mathrm{~m}^{-1} \mathrm{~m}^{-3} \rightarrow$ $9.48 \cdot 10^{22} \mathrm{~m}^{-1} \mathrm{~m}^{-3}$ only). Simultaneous gradual decrease of minimal value of linear growth rate, $G_{0}$, from $3.74 \cdot 10^{-10} \mathrm{~m} \mathrm{~s}^{-1}$ to $1.79 \cdot 10^{-10} \mathrm{~m} \mathrm{~s}^{-1}$ contributes to the advisable, moderate increase of nucleation rate, $B$, value (see Eq. (6)).

\section{CONCLUSIONS}

Integrated processes of precipitation (effect of fast ionic reactions) followed by crystallization of struvite in a continuous liquid-liquid jet-pump DTM $\downarrow$ crystallizer were performed without any disturbances. The product crystals of mean size from ca. 16 to $31 \mu \mathrm{m}$ were removed from the apparatus. It may be expected, that in a DTM $\downarrow$ crystallizer construction an convenient hydrodynamic environment for the effective reduction of generated supersaturation was established, in spite of application of the minimal values of unit power of a jetpump's feeding stream, $P_{\text {eu }}$ (in the range of $0.11-1.45$ $\mathrm{W} \mathrm{kg}{ }^{-1}$ ).

The reader's attention should be also paid on the opposing hydrodynamic effects observed in the two crystallizer constructions under this study. More intensive mixing - observed in a DT crystallizer strongly catalyzes contact nucleation (attrition) but by providing more homogeneous state of circulated solution (or suspension) prevents the unwanted primary nucleation effects. Contrary, the DTM $\downarrow$ construction inhibits contact nucleation (even including external pump contribution - see element (2) in Fig. 1) effectively; however, its moderate circulation intensity is not able to eliminate the bulky zones where primary nucleation can take place.

Increase of circulation intensity in the last construction (DTM $\downarrow$ ) could probably lead to inverse 
the tendencies, thus making it more hydrodynamically similar to a DT-type design. However, actually applied work regime - minimal intensity of circulation - both favours the production of larger crystals (technological advantage) and consumes lower amount of electrical energy (economical advantage), thus can be recommended in the modern technologies of effective phosphorus recycling.

The limiting (lower) values of hydrodynamic $\left(P_{\text {eu }}\right)$ and kinetic $\left(G_{\infty}, G_{0}, B\right)$ parameters of a struvite reaction-crystallization process were identified experimentally. It may be noted, that this minimal value of unit power, being the parameter dependent mainly on physical properties of the two-phase system and geometrical proportions (nozzle diameter - crystallizer volume) - see Eq. (2), can be recognized as an practically useful similarity simplex, relatively easy transferable during eventual scale-up considerations.

Considering the complexity of reactioncrystallization operations the authors used a mathematically convenient MSMPR kinetic model however developed theoretically for classical mass crystallization process - for the evaluation of resulting kinetics without the problematical necessity of considering all possible, detailed interactions between the subprocesses in various scales. This way the resulting kinetic data appearing in the SDG kinetic model presented should be also interpreted as the "effective", "substitute" values, describing globally the overall process kinetics, however very convenient for the design applications, e.g. in a mineral fertilizer industry, providing one with the attractive possibility of altering the product's CSD by indirect influence of selected process parameters.

\section{ACKNOWLEDGMENTS}

This work was supported by the Ministry of Science and Higher Education (of Poland) under grant No. R05 05301.

Crystal size distributions of precipitated struvite were measured by means of particle size analyzer COULTER LS - 230 in the Institute of Inorganic Chemistry, Gliwice, Poland.

The images of struvite crystals (scanning electron microscope JEOL JSM 5800LV) were performed in Head of Materials Science Laboratory of the Institute of Materials Science and Applied Mechanics, Wroclaw University of Technology, Wroclaw, Poland.

Chemical analysis of mother liquor and solid phase compositions were performed in analytical laboratory of the Institute of Inorganic Chemistry in Gliwice, Poland, and in analytical laboratory of the Chemistry for Agriculture Division of the Institute of Inorganic Technology and Mineral Fertilizers of Wroclaw University of Technology, Poland.

\section{REFERENCES}

1. Doyle, J. and S.A. Parsons, 2002. Struvite formation, control and recovery. Wat. Res., 36: 3925 - 3940.

2. Grzmil, B. and J. Wronkowski, 2004. Process for removing and recovering phosphates from wastewaters. Przem. Chem., 83: 275 - 280 (in Polish).

3. Gorazda, K., Z. Wzorek, M. Jodko and A.K. Nowak, 2004. Struvite - manufacturing methods. Chemik, 57: 317 - 320 (in Polish).

4. Parsons, S.A., 2001. Recent scientific and technical developments: struvite precipitation. Scope Newslett., 41: 15 - 22.

5. Booker, N.A., A.J. Pristley and I.H. Fraser, 1999. Struvite formation in wastewater treatment plants: opportunities for nutrient recovery. Environ. Technol., 20: 777 - 782.

6. Bridger, G., 2001. Fertiliser value of struvite. Scope Newslett., 43: 3 - 4 .

7. de-Bashan, L.E. and Y. Bashan, 2004. Recent advences in removing phoshorus from wastewater and its future use as fertilizer. Wat. Res., 38: 4222 4246.

8. Matynia, A., J. Koralewska, K. Piotrowski and B. Wierzbowska, 2006. The influence of process parameters on struvite continuous crystallization kinetics. Chem. Eng. Comm., 193: 160 - 176.

9. Matynia, A., 1997. Crystallizers with a jet pump. Inz. Ap. Chem., 36(6): 9 - 14 (in Polish).

10. Rojkowski, Z. and J. Synowiec, 1991. Crystallization and the crystallizers. WNT, Warszawa (in Polish).

11. Randolph, A.D. and M.A. Larson, 1988. Theory of particulate processes: analysis and techniques of continuous crystallization. Academic Press, New York.

12. Matynia, A., J. Koralewska, B. Wierzbowska and K. Piotrowski, 2005. Jet-pump crystallizers in the reaction-crystallization processes of sparingly soluble salts. Pol. J. Chem. Technol., 7(3): 56-64. 
13. Koralewska, J., A. Matynia, B. Wierzbowska and K. Piotrowski, 2006. Hydroxyapatite crystallization process in a DTM crystallizer of descending suspension flow in a mixing chamber. Pol. J. Chem. Technol., 8(3): $25-27$.

14. Koralewska, J., A. Matynia, K. Piotrowski and B. Wierzbowska, 2006. Crystallization of barium sulphate in a continuous DTM type crystallizer with a jet-pump of descending suspension flow in a mixing chamber. Materials of the International Congress of Chemical and Process Engineering CHISA, CD-ROM, No. 278, Proc. Eng. Publisher, Prague, Czech Republic.

15. Koralewska, J., A. Matynia, K. Piotrowski and B. Wierzbowska, 2006. Precipitation of barium ions with solid ammonium sulphate in a continuous DTM crystallizer with liquid jet-pump of ascending suspension flow in a mixing chamber. Chem. Proc. Eng., 27: 1555 - 1579.

16. Koralewska, J., A. Matynia, B. Wierzbowska and K. Piotrowski, 2006. Recovery of phosphate ions by precipitation and crystallization of struvite in a DTM type crystallizer with a jet-pump of ascending suspension flow in a mixing chamber. Inz. Ap. Chem., 45(5s): 67 - 68 (in Polish).

17. Mullin, J.W., 1993. Crystallization. ButterworthHeinemann, Oxford.

18. Matynia, A., B. Wierzbowska, J. Koralewska, K. Piotrowski, Z. Bechtold and E. Cwiertnia, 2005. DTM-type crystallizers with a jet-pump in reaction-crystallization processes - a novel efficacious approach. Chem. Agricult., 6: 879 886.

19. Bechtold, Z., M. Malasinska, A. Matynia and K. Piotrowski, 2006. Influence of selected jet-pump design parameters on a unit power of feeding stream in a DTM crystallizer with descending suspension flow in a mixing chamber. Chem. Agricult., 7: 371 - 379 .
20. Matynia, A., J. Koralewska and J. Kwiecien, 2003. The influence of the continuous crystallization process parameters on the crystal size distribution of struvite. Pol. J. Chem. Technol., 5(4): 83-89.

21. Koralewska, J., A. Matynia, K. Piotrowski and B. Wierzbowska, 2006. Reaction-crystallization process in a struvite precipitation technology application of a continuous DTM crystallizer with a jet-pump generating descending flow of suspension in a mixing chamber. Chem. Agricult., 7: 380 391.

22. Canning, T.F. and A.D. Randolph, 1967. Some aspects of crystallization theory: Systems that violate McCabe's Delta L Law. AIChE J., 13: 5 10 .

23. Abegg, C.F., J.D. Stevens and M.A. Larson, 1968. Crystal size distribution in continuous crystallizers when growth rate is size - dependent. AIChE J., 14: 118 - 122.

24. Rojkowski, Z., 1977. New empirical kinetic equation of size dependent crystal growth and its use. Kristall und Technik, 12: 1121 - 1128.

25. Rojkowski, Z., 1978. New hyperbolic empirical model of size dependent crystal growth. Bulletin de L'Academie Polonaise des Sciences - Serie des sciences chimiques, 26: $265-270$.

26. Rojkowski, Z., 1978. Two parameter kinetic equation of size dependent crystal growth. Kristall und Technik, 13: 1277 - 1284. 